\title{
On the characteristics of the equations of motion for a bubbly flow and the related problem of critical flow
}

\author{
A. PROSPERETTI* and L. VAN WIJNGAARDEN** \\ California Institute of Technology, Pasadena, California, U.S.A.
}

(Received July 30, 1975)

\begin{abstract}
SUMMARY
For the study of transients in gas-liquid flows, the equations of the so-called separated flow model are inadequate, because they possess, in the general case where gas and liquid move at different velocities, complex characteristics. This paper is concerned with the equations of motion for bubbly flow. The equations are discussed with emphasis on the aspects of relative motion and the characteristics are calculated. It is found that all characteristics are real. The results are used to establish a relation between gas velocity, liquid velocity, void fraction and sound velocity at critical flow. This relation agrees very well with experimental data for these quantities as measured by Muir and Eichhorn in the throat of a converging-diverging nozzle.
\end{abstract}

\section{Introduction}

It is common practice in the literature on gas/liquid flows to use the so-called separated flow model for which separate conservation equations for each of the constituents are written down. When calculating the characteristics of these equations, a difficulty is encountered because it appears that, when a relative velocity between the phases is allowed for, these characteristics are complex. This is discussed e.g. in Wallis [1], Chapter 6. A lively debate on this subject took place during a Round Table Discussion held at the 5th International Heat Transfer Conference in Tokyo, 1974 [2]. The fact that characteristics are complex makes the Cauchy problem for these equations an ill posed problem. Therefore the separated flow model does not seem to be fully adequate for the investigation of wave motion in two phase flows. The physical reason for the complex characteristics presumably is, (see the above mentioned Round Table Discussion) that instabilities of the interface like Kelvin-Helmholtz instability are hidden in the model. This means that a initially separated flow turns under certain conditions into another topology. In many cases this new topology is a dispersed flow in which the gas is dispersed as bubbles in the liquid. With a view to problems such as transients in two phase flows in nuclear reactors, it is of some interest to consider the equations of motion of a bubbly flow, in particular when the relative velocity is appreciable. In this paper we formulate these equations and calculate their characteristics. We assume that the pressure in the bubbles equals the local pressure in the liquid. This assumption is almost always made in the modeling of gas/liquid flows. Further we assume that the bubbles behave isothermally. Some evidence in support of the validity of

\footnotetext{
* On leave of absence from Istituto di Fisica, Universitá Degli Studi, Milano, Italy.

$\star \star$ Permanent address: Twente Institute of Technology, Enschede, The Netherlands.
} 
the model is provided by the favorable comparison with experiment of the critical velocity derived from our theory.

\section{Equations of motion}

We consider time-dependent flow in the $x$ direction of a mixture of liquid (density $\rho_{l}$, velocity $u$ ) and gas bubbles (density $\rho_{g}$, velocity $v$ ). Locally the bubbles have all the radius $a$. An extension to a distribution of bubble sizes is possible; here we shall restrict to one bubble size, locally. If the void fraction, that is the concentration of gas by volume, is denoted by $\alpha$ and the number density by $n$, we have

$$
\alpha=\frac{4}{3} \pi a^{3} n=n \tau,
$$

where $\tau$ is the volume of a bubble at position $x$ and time $t$. For most practical applications we can safely neglect the contribution of the gas to the density of the mixture, because this is significant only for $\alpha \approx 1$. Denoting the density of the mixture by $\rho$, we have therefore

$$
\rho=\rho_{l}(1-\alpha) \text {. }
$$

The pressure $p_{g}$ of the gas in the bubbles is not necessarily equal to the pressure $p$ in the surrounding liquid, nor need the temperatures be equal. For acoustic waves in liquidbubble mixtures differences in pressure and temperature give rise to dispersion and damping (see e.g. van Wijngaarden [3]). The importance of these effects is small when the characteristic times of the flow are large compared to the natural period of oscillation of the bubbles and to the relaxation time. In the present work we want to investigate the implications of relative translational motion between bubbles and liquid and therefore we make the most simplifying assumptions where other effects are concerned, viz $p_{g}$ equals $p$ and isothermal bubble behavior. It should be noticed that even in the presence of dispersion and diffusion, to a first approximation the waves are centered about the characteristics determined here. Since both $u$ and $v$ are involved in the formulation of the equations of motion, we have two material derivatives,

$$
\frac{d}{d t}=\frac{\partial}{\partial t}+u \frac{\partial}{\partial x}
$$

and

$$
\frac{D}{D t}=\frac{\partial}{\partial t}+v \frac{\partial}{\partial x}
$$

We assume that the bubbles preserve their identity and do not break up or coalesce, which means that the number density $n$ satisfies the conservation equation

$$
\frac{D n}{D t}+n \frac{\partial v}{\partial x}=0 \text {. }
$$

For an isothermal bubble we have in addition

$$
\frac{D}{D t}(p \tau)=0
$$


which gives together with (1) and (5)

$$
\frac{D}{D t}(p \alpha)+p \alpha \frac{\partial v}{\partial x}=0
$$

Taking the liquid as incompressible, mass conservation requires with use of (2),

$$
\frac{d}{d t}(1-\alpha)+(1-\alpha) \frac{\partial u}{\partial x}=0
$$

If $v$ were equal to $u,(7)$ and (8) would give

$$
\frac{D}{D t} \frac{p \alpha}{1-\alpha}=0
$$

which can be regarded as an equation of state and from which a sound velocity, $c_{0}$ say, follows as

$$
c_{0}^{2}=\frac{D p}{D \rho}=\frac{p}{\rho_{l} \alpha(1-\alpha)} .
$$

Now that $v \neq u$, no relation $p=p(\rho)$ exists. From (6)-(8) it follows that

$$
\frac{D}{D t} \frac{p \alpha}{1-\alpha}=-\frac{p \alpha}{(1-\alpha)^{2}} \frac{\partial}{\partial x}\{(1-\alpha)(v-u)\}
$$

The derivative in the right hand side cannot be written as a material derivative of a function containing $p$ and $\alpha$ only.

The equation expressing conservation of momentum is

$$
\rho \frac{d u}{d t}+\frac{\partial p}{\partial x}=0
$$

with $\rho$ given by (2). To the equations (7), (8) and (10) an equation for the relative motion must be added. We assume that the Reynolds number for the relative motion around an individual bubble is large enough for inertia forces to dominate over viscous forces. Then the flow, to a first approximation in the reciprocal Reynolds number, can be calculated with potential theory and the resistance force is given by $-k \mu a(v-u)$, where $k$ is a constant ( $12 \pi$ for a sphere) and $a$ is the effective radius of the bubble, whereas $\mu$ is the dynamic viscosity of the liquid (see Landau and Lifshitz [4] and Levich [5]). Since we have potential flow around the bubble, we can apply the theorem (see e.g. Landau and Lifshitz [4]) that the rate of change of the liquid impulse, given by the product of relative velocity and virtual mass, equals the external force on the bubble. This includes the resistance force, mentioned above, and in addition the inertia force $\rho_{l} \tau(D u / D t)$. Note that the latter is not $\rho_{l} \tau(d u / d t)$, because it is the acceleration of the liquid observed in a frame moving with the bubbles that matters. How to calculate the virtual mass of a body in an unbounded liquid is treated in almost any textbook on hydrodynamics. However it is more complicated in the present case where the virtual mass of any individual bubble will be affected by the presence of the 
other bubbles. For spherical bubbles and small $\alpha$ this dependence on other bubbles can according to van Wijngaarden [6] be expressed by, $m$ representing the virtual mass,

$$
m=\lambda \rho_{l} \tau,
$$

where

$$
\lambda=\frac{1}{2}(1+2.78 \alpha)+O\left(\alpha^{2}\right) .
$$

We shall use (11) and (12) in the following. Then the equation for the relative motion of an individual bubble is

$$
\frac{D}{D t} \lambda \rho_{l} \tau(v-u)=\rho_{l} \tau \frac{D u}{D t}-k \mu a(v-u) .
$$

Upon multiplication of this equation with $n$ and of (5) with $\lambda \rho_{l} \tau(v-u)$, addition of the results gives with the help of (10)

$$
\begin{aligned}
& \frac{D}{D t} \alpha(v-u)+\frac{\alpha}{\lambda}(v-u) \frac{D \lambda}{D t}+\alpha(v-u) \frac{\partial v}{\partial x} \\
& \quad+\frac{\alpha}{\rho_{l} \lambda(1-\alpha)} \frac{\partial p}{\partial x}-\frac{\alpha(v-u)}{\lambda} \frac{\partial u}{\partial x}=-\frac{k n v a(v-u)}{\lambda}
\end{aligned}
$$

where $v$ is the kinematic viscosity of the liquid. In order to simplify we write in the term for the resistance force $n a$ as $n a^{3} / \tilde{a}^{2}$, where $\tilde{a}$ is a representative average bubble radius and define the relaxation time $T$ as

$$
T=\frac{4 \pi \lambda \tilde{a}^{2}}{3 k v} \text {. }
$$

$T$ is a measure for the time which it takes the liquid to decelerate by viscous forces a faster moving bubble to the liquid velocity. With this the equation for relative motion finally takes the form, $\lambda^{\prime}$ denoting $d \lambda / d \alpha$,

$$
\begin{gathered}
\frac{D}{D t} \alpha(v-u)+\frac{\alpha \lambda^{\prime}}{\lambda}(v-u) \frac{D \alpha}{D t}+\alpha(v-u) \frac{\partial v}{\partial x}+\frac{\alpha}{\rho_{l} \lambda(1-\alpha)} \frac{\partial p}{\partial x} \\
-\frac{\alpha(v-u)}{\lambda} \frac{\partial u}{\partial x}=-\frac{\alpha(v-u)}{T} .
\end{gathered}
$$

The set of equations (7), (8) and (15) still describe the motion of a homogeneous liquid. As compared with the case $u=v$ there is now however an additional internal degree of freedom, viz. $(v-u)$. This interpretation is quite fruitful as shown by Noordzij and van Wijngaarden [7] in their study of the effect of relative motion on shock waves.

\section{Characteristics}

It is convenient to use as dependent variables $\alpha, u, V$ and $p$ where

$$
V=v-u \text {. }
$$


Rewritten in terms of this variable (8), (10), (7) and (15) become, in that order,

$$
\begin{aligned}
& -\frac{D \alpha}{D t}+V \frac{\partial \alpha}{\partial x}+(1-\alpha) \frac{\partial u}{\partial x}=0, \\
& \rho_{l}(1-\alpha) \frac{D u}{D t}-\rho_{l}(1-\alpha) V \frac{\partial u}{\partial x}+\frac{\partial p}{\partial x}=0, \\
& p \frac{D \alpha}{D t}+\alpha \frac{D p}{D t}+\alpha p \frac{\partial u}{\partial x}+\alpha p \frac{\partial V}{\partial x}=0, \\
& \left(1+\frac{\alpha \lambda^{\prime}}{\lambda}\right) V \frac{D \alpha}{D t}+\alpha \frac{D V}{D t}+\alpha V \frac{\partial u}{\partial x}+\alpha V \frac{\partial V}{\partial x}+\frac{\alpha}{\rho_{l}(1-\alpha) \lambda} \frac{\partial p}{\partial x} \\
& \quad-\frac{\alpha V}{\lambda} \frac{\partial u}{\partial x}=-\frac{\alpha V}{T} .
\end{aligned}
$$

To obtain the characteristics of the set of equations (17)-(20) we write

$$
\frac{D}{D t}=-\sigma \frac{\partial}{\partial x},
$$

and find upon substitution of this into (17)-(20) that the $\sigma$ 's are given by

$$
\left|\begin{array}{cccc}
\sigma+V & 1-\alpha & 0 & 0 \\
0 & -\rho_{l}(1-\alpha)(\sigma+V) & 0 & 1 \\
-\sigma p & \alpha p & \alpha p & -\alpha \sigma \\
-\left(1+\frac{\alpha \lambda^{\prime}}{\lambda}\right) \sigma V & \alpha V\left(1-\frac{1}{\lambda}\right) & \alpha(V-\sigma) & \frac{\alpha}{\lambda \rho_{l}(1-\alpha)}
\end{array}\right|=0 .
$$

Before going on, we render $\sigma$ and $V$ dimensionless with the velocity $c_{0}$, defined in (9),

$$
\begin{aligned}
& \frac{V}{c_{0}}=\varphi, \\
& \frac{\sigma}{c_{0}}=\xi,
\end{aligned}
$$

Moreover we define

$$
\begin{aligned}
& \beta=1+\lambda^{-1}+(1-\alpha) \lambda^{-1} \lambda^{\prime}, \\
& \gamma=1+\alpha \lambda^{-1} .
\end{aligned}
$$

Using (23)-(26), we obtain from (22)

$$
\xi\left\{\xi(\xi+\varphi)^{2}-\varphi\left[\alpha \beta+(\xi+\varphi)^{2}\right]-\gamma \xi\right\}=0 .
$$

Obviously one of the roots is $\xi=0$, corresponding to

$$
\frac{D}{D t}=0
$$


The three other ones are the roots of the cubic equations in the curly brackets in (27). In order to gain some insight into the nature of these characteristics we calculate these for the practically important case where $\varphi$ is a small quantity. Writing

$$
\xi=\xi_{0}+\varphi \xi_{1}+\varphi^{2} \xi_{2}+\ldots
$$

we obtain, neglecting terms of order $\varphi^{2}$,

$$
\xi_{0}^{3}+3 \varphi \xi_{0}^{2} \xi_{1}+\varphi \xi_{0}^{2}-\gamma \bar{\zeta}_{0}-\gamma \varphi \xi_{1}-\alpha \beta \varphi=0 .
$$

Collecting terms of the same order in $\varphi$, this gives

$$
\xi_{0}=0, \text { with } \xi_{1}=-\frac{\alpha \beta}{\gamma},
$$

and

$$
\xi_{0}^{2}=\gamma, \text { with } \xi_{1}=-\frac{1}{2}+\frac{\alpha \beta}{2 \gamma} .
$$

Returning to dimensional variables we have for the characteristics $d x / d t$, from these expression and (4), (21), (23) and (24), up to the second order in $(v-u) / c_{0}$,

$$
\begin{aligned}
& \left(\frac{d x}{d t}\right)_{1}=v, \\
& \left(\frac{d x}{d t}\right)_{2}=v-\frac{\alpha \beta}{\gamma}(v-u), \\
& \left(\frac{d x}{d t}\right)_{3,4}=\frac{1}{2}(v+u)+\frac{1}{2} \alpha \frac{\beta}{\gamma}(v-u) \pm c_{f},
\end{aligned}
$$

where

$$
c_{f}=\gamma^{\frac{1}{2}} c_{0} .
$$

$c_{f}$ is the speed of sound in a mixture in which relative motion is not resisted by viscous friction (see e.g. van Wijngaarden [3]), whereas $c_{0}$ is the speed of sound in a mixture in which viscous forces exerted on the bubbles are strong enough to prevent relative motion. It is completely in agreement with the theory of nonequilibrium flow that in the general case in which $T$, in equation (15), is neither infinite nor zero, $c_{f}$ emerges in the expression for the characteristics, as here in (31) (see e.g. Wegener [8]). It appears that the terms containing $(v-u)$ in (30) and (31) are preceded by $\alpha$. Hence when both $\alpha$ and $(v-u) / c_{0}$ are small and of the same order of smallness, these terms can be neglected and in that case (29)-(31) reduce further to

$$
\begin{aligned}
& \left(\frac{d x}{d t}\right)_{1,2}=v \\
& \left(\frac{d x}{d t}\right)_{3,4}=\frac{u+v}{2} \pm c_{f} .
\end{aligned}
$$

Particularly interesting are the two characteristics (34), corresponding to the well known 
" $u \pm c$ " characteristics in the dynamics of single phase flow. It could be anticipated that the convective part would contain both $u$ and $v$. However, it is rather surprising that, in the approximation underlying (34), the convective part in the characteristic speed is just the arithmetic mean of these two velocities. It should be emphasized that this result holds for bubbles of any shape, because the shape enters only in the higher order terms, which contain the factor $\lambda$.

The results of this section show that the system of equations (17)-(20) is completely hyperbolic, so that for these equations the Cauchy problem is a well posed one.

\section{Application to critical flow. Comparison with experiment}

When a single phase flow expands through a Laval nozzle under conditions where the reservoir pressure is kept constant but the exit pressure is progressively decreased from the reservoir pressure, a maximum in the mass flow from the nozzle occurs when in the throat the velocity equals the velocity of sound. This so-called critical flow occurs in two phase flows as well and is of technological importance, but the interpretation is less simple than in single phase flow because of the different velocities of the phases. For bubbly flows obeying equations (17)-(20), we can now predict critical flow from the criterion that in that case a stationary wave in the throat is possible, because then a disturbance cannot travel upstream from the throat. From (4) and (21) it follows that critical flow occurs when

$$
\sigma=-v \text {. }
$$

Using (23) and (24) we find upon inserting this into the cubic equation in the square bracket in (27) and returning to the variables $u$ and $v$,

$$
c_{0}^{2}=\frac{2 u^{2} v-u^{3}}{\alpha \beta u+(\gamma-\alpha \beta) v}
$$

It can be readily verified that in case $(v-u) / c_{f}$ is a small quantity, this reduces to

$$
\frac{1}{2}(v+u)+\frac{1}{2} \alpha \frac{\beta}{\gamma}(v-u)=c_{f},
$$

which also follows from (31) by putting $(d x / d t)_{4}$ equal to zero. Equation (35) gives a relation between $u, v, \alpha$ and $c_{0}$ which should with reasonable accuracy hold in the throat of a Laval nozzle. Because of friction the effective throat is always located a little downstream from the geometrical throat, but usually this is a relatively small effect. Experiments with bubbly flows through Laval nozzles have been reported by Muir and Eichhorn [9]. They measured $u, v, c_{0}$ and $\alpha$ in the throat and their data provide us with an opportunity to verify equation (35). In Table 1 we have collected the values of $u, v$ and $c_{0}$ for different values of $\alpha$, as read from figure 15 in reference 9 . The values of $\left(c_{0}\right)_{\text {theor. }}$, which follow from equation (35) for the same $u, v$, and $\alpha$ are also given in the same Table for comparison. In the last column of Table 1 the relative difference with the experimental value $\left(c_{0}\right)_{\exp }$ is given. It appears that this difference is of the order of $10 \%$ and further that systematically the theoretical value is smaller than the experimental one. This may be attributed to the fact that the flow in the geometrical throat is not yet critical: 
TABLE 1

Critical flow conditions. Comparison between the data of Figure 15 of reference 9 and the present theoretical result, equation (35). Velocities are expressed in $\mathrm{m} / \mathrm{sec}$.

\begin{tabular}{llllll}
\hline & $u$ & $v$ & $c_{0, \exp }$ & $c_{0}, \mathrm{th}$ & $\frac{c_{0, \exp }-c_{0}, \mathrm{th}}{c_{0, \exp }}, \%$ \\
\hline 0.08 & 33.2 & 40.2 & 38.2 & 34.9 & 8.6 \\
0.11 & 31.1 & 37.5 & 33.7 & 32.3 & 4.2 \\
0.16 & 25.0 & 31.1 & 29.6 & 26.1 & 11.8 \\
0.18 & 23.5 & 29.6 & 27.7 & 24.6 & 11.2 \\
0.22 & 21.3 & 27.7 & 25.9 & 22.7 & 12.3 \\
0.25 & 20.1 & 26.8 & 24.4 & 21.6 & 11.5 \\
0.30 & 18.9 & 25.3 & 23.2 & 20.3 & 12.5 \\
0.33 & 17.7 & 25.0 & 22.3 & 19.6 & 12.1 \\
\hline
\end{tabular}

It can be concluded that the agreement of the present theory with the experiments of Muir and Eichhorn is satisfactory.

\section{Characteristic form of the equations}

In view of the hyperbolic nature of the system (17)-(20) it is of some interest to put the equations in characteristic form. This may be useful for numerical computation, and may also lead to the identification of conserved quantities in special cases. In this connection it is particularly interesting to determine whether the analogues of the Riemann invariants of gas dynamics can be constructed for our system. It will be shown that this is not possible in the general case.

In terms of $v$ (rather than $V$ ) the system (17)-(20) can be written in matrix form as

$$
A \frac{\partial \Gamma}{\partial t}+B \frac{\partial \Gamma}{\partial x}=\Delta
$$

where $A=\left(a_{i j}\right)$ and $B=\left(b_{i j}\right)$ are the matrices

$$
\begin{aligned}
& A=\left|\begin{array}{llll}
-1 & 0 & 0 & 0 \\
0 & \rho_{l}(1-\alpha) & 0 & 0 \\
P & 0 & 0 & \alpha \\
V\left(1+\alpha \lambda^{\prime} / \lambda\right) & -\alpha & \alpha & 0
\end{array}\right| \\
& B=\left|\begin{array}{llll}
-u & 1-\alpha & 0 & 0 \\
0 & \rho_{l}(1-\alpha) u & 0 & 1 \\
v p & 0 & p \alpha & \alpha v \\
v V\left(1+\alpha \lambda^{\prime} / \lambda\right) & -\alpha[v+V / \lambda] & \alpha(V+v) & \alpha /\left[\rho_{l}(1-\alpha) \lambda\right]
\end{array}\right|
\end{aligned}
$$

and the column vectors $\Gamma=\left(\gamma_{i}\right), \Delta=\left(\delta_{i}\right)$ are defined as

$$
\Gamma^{T}=(\alpha, u, v, p), \quad \Delta^{T}=(0,0,0,-\alpha V / T)
$$


with the superscript $T$ denoting the transpose. Now we wish to form linear combinations of the equations (36) such that in each equation all the quantities are differentiated along the same direction. To this end we multiply the left-hand side of (36) by a row vector $L^{T}=\left(l_{i}\right)$ and require that

$$
\sum_{i=1}^{4} l_{i}\left(a_{i j} \frac{\partial \Gamma_{j}}{\partial t}+b_{i j} \frac{\partial \Gamma_{j}}{\partial x}\right) \equiv \sum_{k=1}^{4} m_{k}\left(\frac{\partial \Gamma_{k}}{\partial t}+C \frac{\partial \Gamma_{k}}{\partial x}\right)
$$

where the quantities $l_{i}, m_{k}$, and $C$ are to be determined. From (37) it follows that

$$
\sum_{i=1}^{4} l_{i} a_{i j}=m_{j}, \quad \sum_{i=1}^{4} l_{i} b_{i j}=C m_{j}
$$

or, eliminating $\mathrm{m}_{j}$

$$
\sum_{i=1}^{4} l_{i}\left(C a_{i j}-b_{i j}\right)=0, \quad j=1,2,3,4 .
$$

It is well known that the condition of compatibility for this linear homogeneous system determines the characteristic directions, and indeed it is easily shown that the condition $\operatorname{det}\left|C a_{i j}-b_{i j}\right|=0$ is equivalent to Eq. (22) with $C=\sigma+v$, in agreement with (21). In terms of $C$, Eq. (27) is

$$
\begin{gathered}
(C-v)\left\{C^{3}-(u+2 v) C^{2}+\left(4 u v-u^{2}-\gamma c_{0}^{2}\right) C+\right. \\
\left.+u^{3}-2 u^{2} v+[\alpha \beta u+(\gamma-\alpha \beta) v] c_{0}^{2}\right\}=0 .
\end{gathered}
$$

Solving now (39) for the $l_{i}$ 's and determining the $m_{j}$ 's from either one of Eqs. (38), it is readily proven that the system (36) is equivalent to the following four equations

$$
\begin{aligned}
& p V\left(C_{j}-v-\alpha V \lambda^{\prime} \lambda^{-1}\right) D_{j} \alpha+p \alpha\left(C_{j}-u\right) D_{j} v \\
& \quad+\alpha\left(C_{j}-u\right)\left[\rho_{l}(1-\alpha)\left(C_{j}-v\right)\left(C_{j}-v-V\right)-p\left(1+\lambda^{-1}\right)\right] D_{j} u \\
& \quad+\alpha\left(C_{j}-u\right)\left(C_{j}-v-V\right) D_{j} p=-\alpha T^{-1} V p\left(C_{j}-u\right), \quad j=1,2,3,4,
\end{aligned}
$$

where

$$
D_{j}=\frac{\partial}{\partial x}+C_{j} \frac{\partial}{\partial x}
$$

with the $C_{j}$ 's the four roots of the characteristic equation (40). The first equation of the system (41), corresponding to the root $C_{1}=v$, is nontrivial only if $v \neq u$ and is

$$
-p V \lambda^{\prime} \lambda^{-1} D_{1} \alpha-p\left(1+\lambda^{-1}\right) D_{1} u+p D_{1} v-V D_{1} p=-T^{-1} p V
$$

which can also be written more compactly as

$$
\lambda^{\prime} \lambda^{-1} V D_{1} \alpha+\lambda^{-1} D_{1} u+p D_{1}(V / p)=-T^{-1} V .
$$

It is evident that in general one cannot find a function $R(\alpha, u, v, p, x, t)$ such that this equation can be put into the form

$$
\frac{d R}{d t}=f(\alpha, u, v, p, x, t) \text { on } \frac{d x}{d t}=v
$$


and indeed it is very easy to give a formal proof of this fact. The non-existence of the function $R$ in general precludes the possibility of finding Riemann invariants (or rather Riemann variables) for the entire system (41).

In view of the complicated analytic expression of the other three roots of (40) it is not possible to put the remaining equations of the system (41) in as simple a form as that of Eq. (42) or (43). However, the integration of (41) along the characteristic directions can be performed numerically.

\section{Acknowledgement}

This study was supported in part by the Electric Power Research Institute, Palo Alto, California, USA.

\section{REFERENCES}

[1] G. B. Wallis, One-dimensional two-phase flow, McGraw-Hill, New York (1969).

[2] Modeling of two-phase flow. Round Table Discussion RT-1-2, 5th Int. Heat Transfer Conf. Tokyo, (1974).

[3] L. van Wijngaarden, One-dimensional flow of liquids containing small gas bubbles, Ann. Rev. Fluid Mech., 4 (1972) 369.

[4] L. D. Landau and E. M. Lifshitz, Fluid Mechanics, Pergamon Press (1959).

[5] V. G. Levich, Physicochemical Hydrodynamics, Prentice-Hall (1962).

[6] L. van Wijngaarden, Hydrodynamic interaction between bubbles in a dilute gas bubble/liquid mixture; to be published.

[7] L. Noordzij and L. van Wijngaarden, Relaxation effects, caused by relative motion, on shock waves in gas-bubble liquid mixtures, J. Fluid Mech., 66 (1974) 1.

[8] P. P. Wegener, Nonequilibrium Flow, vol. II, Marcel Dekker, New York (1970).

[9] J. F. Muir and R. Eichhorn, Compressible flow of an air-water mixture through a vertical, two-dimensional, converging-diverging nozzle, Proc. of the 1963 Heat Transfer and Fluid Mechanics Institute, Stanford Univ., Stanford (1963). 